\title{
Two-Dimensional AMR-PIC Plasma Simulation for Mini-Magnetosphere of Magnetized Object ${ }^{*}$
}

\author{
Masaharu MATSUMOTO ${ }^{\text {a) }}$, Hideyuki USUI, Masanori NUNAMI ${ }^{1)}$, \\ Masao NAKAMURA ${ }^{2)}$ and Iku SHINOHARA ${ }^{3)}$ \\ Kobe University, 1-1 Rokkodai-cho, Nada-ku, Kobe 657-8501, Japan \\ 1) National Institute for Fusion Science, 322-6 Oroshi-cho, Toki 509-5292, Japan \\ ${ }^{2)}$ Osaka Prefecture University, 1-1 Gakuen-cho, Naka-ku, Sakai 599-8531, Japan \\ 3) Japan Aerospace Exploration Agency, 3-1-1 Yoshinodai, Chuo-ku, Sagamihara 252-5210, Japan
}

(Received 7 December 2012 / Accepted 1 August 2013)

\begin{abstract}
A parallelized two-dimensional full particle-in-cell (PIC) simulations with an adaptive mesh refinement (AMR) technique for the interaction of solar wind with kinetic scale magnetosphere around a magnetic sail are conducted. For multi-scale simulations, the newly developed AMR-PIC code has a method to modify the grid size and time step interval of computational domain in the midst of the simulations temporally and locally. In the simulation results under the typical working condition introducing the AMR technique, it is shown that the direction of interplanetary magnetic field depend strongly on the magnetospheric configuration including electron dynamics and Alfven bow shock structure. Although more validation studies are needed, the AMR-PIC code shows the worth of development for multi-scale simulations.
\end{abstract}

(C) 2013 The Japan Society of Plasma Science and Nuclear Fusion Research

Keywords: kinetic scale magnetosphere, particle-in-cell, adaptive mesh refinement, solar wind, space plasma

DOI: $10.1585 /$ pfr.8.2406132

\section{Introduction}

Magnetic sail is an advanced space propulsion system [1] which uses the interaction between an artificial magnetosphere around a spacecraft and solar wind plasmas. The solar wind is deflected in the magnetosphere and the momentum transfer results in the drag force exerted on the spacecraft. Recently, the Mini-Magnetospheric Plasma Propulsion (M2P2) [2], which is also called MagnetoPlasma Sail (MPS) [3], is suggested by Winglee et al. as a derivative system from Magnetic sail. In the concept of M2P2 and MPS, the original magnetic field attached to a spacecraft is expanded by the injection of artificial plasmas from the spacecraft to form finally a huge magnetosphere. Such propulsion systems which use the solar wind energy are expected to achieve high energy conversion efficiency compared with conventional electric propulsion systems and many feasibility studies have been conducted.

Fujita [4] has revealed that the drag (thrust) force of a magnetic sail, which is exerted on the spacecraft from the solar wind, is characterized by the ratio of an ion Larmor radius of the solar wind at the magnetopause $r_{\mathrm{L}}$ (which is equal to ion inertial length in solar wind) to a representative length of magnetic field $L$. Figure 1 shows the schematic view of typical ion particle motions due to the

author'se-mail: matsumoto@cc.u-tokyo.ac.jp

a) present address: Information Technology Center, The University of Tokyo, 5-1-5 Kashiwanoha, Kashiwa-shi, Chiba 277-8589, Japan

*) This article is based on the presentation at the 22nd International Toki Conference (ITC22). difference in $r_{\mathrm{L}} / L$. On the magnetohydrodynamics (MHD) scale $\left(r_{\mathrm{L}} / L \ll 1\right)$, the ion particle motions depend strongly on the magnetic field line and the momentum of ion particles turn into the drag force of the spacecraft as shown in Fig. 1. On the other hand, on smaller than the ion inertial scale $\left(r_{\mathrm{L}} / L \gg 1\right)$, the electromagnetic interactions of ion particles with magnetic field become weak compared with the case of MHD scale because ion kinetic effect becomes dominant. Consequently, the thrust performance of magnetic sail significantly decreases on such a kinetic scale. In the propulsion system of magnetic sail, at least the magnetospheric size of ion inertial scale or more is needed to obtain the thrust performance higher than conventional electric propulsion systems. Therefore, kinetic

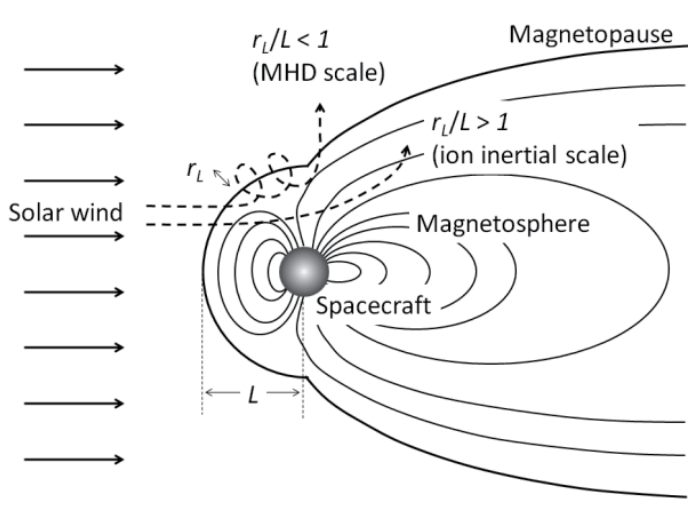

Fig. 1 Ion particle motion due to the difference on $r_{\mathrm{L}} / L$. 
approaches which address the small-scale physical mechanism must be used to simulate the magnetic sail with such small magnetosphere of ion and electron inertial scale.

In the past study of magnetic sail, hybrid particle-incell (PIC) simulation methods have been conducted mainly in order to examine the solar wind flow field around magnetic sail and the thrust performance $[4,5]$. The hybrid PIC simulations, in which ions are treated as super particles and electrons are assumed as fluid, can include the kinetic effect of ion particles. However, the solar wind flow field and thrust performance on the smaller magnetospheric scale than ion inertial, namely, electron inertial scale, have not been almost revealed. This is because the physical phenomena on electron inertial scale cannot be simulated by the hybrid PIC simulation method. On the other hand, full PIC plasma simulation methods [6], in which both electrons and ions are treated as super particles, are widely used in space plasma science and engineering. This method includes the kinetic effect of not only ion but also electron particles. However, the computational load of full PIC simulation is extremely higher than hybrid PIC or MHD simulations because the grid width of computational domain should be set to plasma characteristic lengths such as Debye length and Larmor radius. Furthermore, over one hundred plasma particles per unit cell (grid) are needed for high accuracy simulations.

Our research group has developed an adaptive mesh refinement PIC (AMR-PIC) simulation code [7] in order to handle the problems as mentioned above. The AMR-PIC code is PIC simulation code which has method to modify the grid width of computational domain in the midst of the simulations temporally and locally as shown in Fig. 2. The grid width is adjusted automatically in accordance with the physical values of intended plasma flow field. The AMRPIC method can simulate the physical phenomena in which more resolution is needed, and can decrease the computational load such as memory and computing time compared to conventional PIC code with uniform grid width of computational domain.

Based on the above, the purpose of this study is to

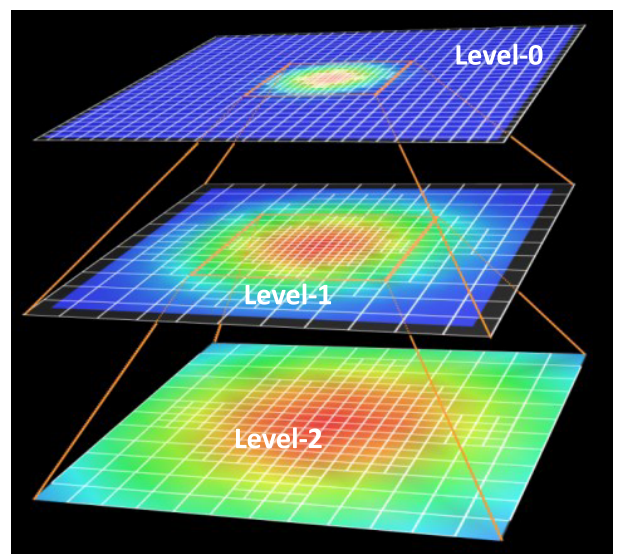

Fig. 2 Grid system of adaptive mesh refinement. examine the interaction of solar wind flow field and kinetic scale magnetosphere around a magnetic sail by using AMR-PIC simulation codes. In the present paper, for the preliminary simulations by the AMR-PIC code, Alfven shock wave configurations which appear at upstream of a magnetic sail are focused under the condition of a kinetic scale magnetosphere.

\section{AMR-PIC Plasma Simulations}

The spatial grid size and time step interval of a full PIC simulation are basically determined by the Debye length, which implies a characteristic spatial length for the electrostatic shielding of a charged particle, and the Courant condition which gives the upper limit of the time step interval for the calculation to avoid the numerical instability. To achieve an efficient simulation with reasonable cost of computer resources, the spatial and temporal resolutions can be adjusted locally and dynamically depending on the local scales of phenomena. To realize this efficiency in PIC simulations, we decided to incorporate the adaptive mesh refinement technique which has been used in the field of computational fluid dynamics as a useful method to investigate such multi-scale phenomena.

The AMR simulation is structured hierarchically. Grid size and time step intervals are defined according to the hierarchy levels, where high and low levels correspond to the fine and coarse grid systems, respectively (Fig. 2). As the simulation system evolves, some complex microscale phenomena can locally and intermittently occur in a hierarchical domain (Level L). If the grid size in Level L is too coarse to simulate the local complex phenomena, a higher hierarchical domain (Level $\mathrm{L}+1$ ) is adaptively created in which the grid spacing size and the time step interval become half of those used in the domain of (Level L). In the Level L+1 domain, to synchronize the temporal advancement to Level L, the number of particle loops for updating the velocities and positions becomes double compared to that in the Level L domain because the time step interval is half in the Level L+1. In the AMR simulation, grids with different spacing are dynamically created in hierarchical layers according to the local conditions (for example, Debye length, Larmor radius, and so on) of phenomena. Fine grids suitable to the local high density region are applied only there and other regions are simulated by using moderate size grids. Therefore, increment of the numerical cost due to the localized region is not serious by adopting the AMR technique. The AMR technique has been rarely employed in PIC codes except for a few examples [8].

\section{Simulation Conditions}

Table 1 and Fig. 3 show the working conditions and computational domain used in this study. In Table $1, m$, $q, T, \omega_{\mathrm{c}}, \omega_{\mathrm{p}}, \beta$ and $M_{\mathrm{A}}$ are static mass, electric charge, temperature, cyclotron frequency, plasma frequency, ratio of static pressure to magnetic pressure and Alfven Mach 
Table 1 Working conditions.

\begin{tabular}{cc}
\hline \hline Total hierarch for AMR & 4 (Lv.0-3) \\
Number of Super Particles & 128 pair/cell@Lv.2 \\
$m_{\mathrm{i}} / m_{\mathrm{e}}$ & 25 \\
$q_{\mathrm{i}} / q_{\mathrm{e}}$ & 1.0 \\
$T_{\mathrm{i}} / T_{\mathrm{e}}$ & 1.0 \\
$\omega_{\mathrm{ce}} / \omega_{\mathrm{pe}}$ & 0.5 \\
$\beta_{\mathrm{i}}, \beta_{\mathrm{e}}$ & 1.0 \\
$M_{\mathrm{A}}$ & 2.0 \\
$r_{\mathrm{L}} / L$ & 4.0 \\
Process and thread number & $\mathrm{Up} \mathrm{to}(\mathrm{p}) 64 \times(\mathrm{t}) 32$ \\
for parallel computing & $=2048$ \\
\hline \hline
\end{tabular}

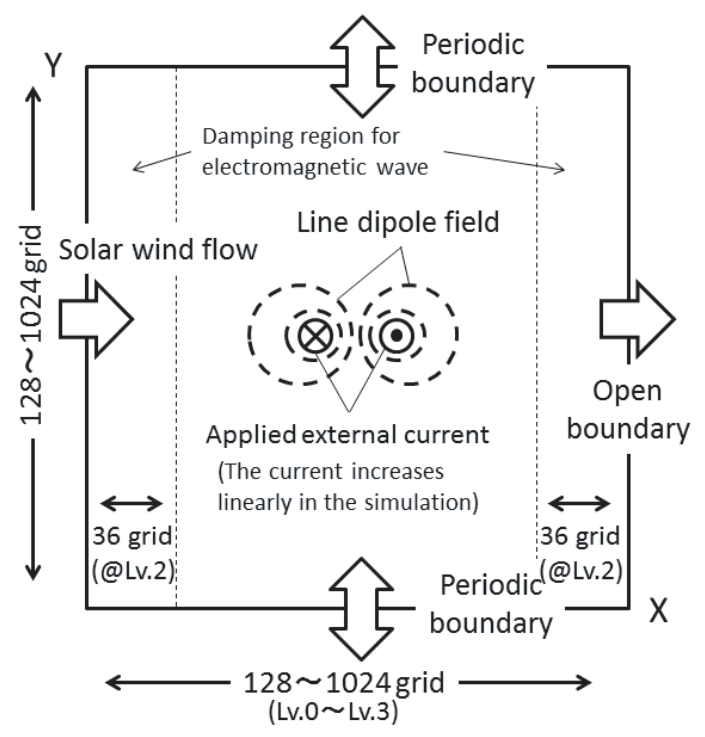

Fig. 3 Computational domain used in this study.

number in solar wind, respectively. Subscript $i$ and e indicate ions and electrons. The scale parameter is set to the kinetic inertial scale $\left(r_{\mathrm{L}} / L=4.0\right)$ although some solar wind parameters are set to arbitrary suitable for the kinetic scale simulation. Total hierarchy for AMR level set to 4 (which is the layer from Lv.0 to Lv.3). The base grid, which is specified by the Debye length of solar wind at upstream, is set to Lv.2. That is, all spatial grids are set to Lv.2 layer at simulation start and some local regions change in corresponding to the fine (Lv.3) and coarse grids (Lv.1 or 0) according to the local Debye length and Larmor radius. The criterions for mesh refinement are set when the electron number density exceeds a critical limit in the computational domain.

Here, the physical parameters assumed in this study are quite different from those of real space plasmas. Although we focus on the kinetic scale magnetosphere $\left(r_{\mathrm{L}} / L\right.$ is of the order of unity) in the present study, the simulations with real parameters are difficult in terms of the computational cost. Therefore the dimensionless parameters are used here as shown in Table 1. We can discuss the kinetic scale magnetosphere qualitatively under such work- ing conditions. The simulations with parameters of real space plasmas are future works.

The solar wind plasma flows from left to right direction and the dipole magnetic field is set on the center of the computational domain in the figure. The line dipole field is excited by the applied current as a magnetic sail which increases linearly in the midst of the simulation. The damping regions for electromagnetic waves are set to the left and right boundary of the computational domain and top and bottom boundary are periodic condition. We use supercomputer (CRAY XE6) of KDK in RISH, Kyoto University and the process and thread number for parallel computing are up to 2048 (64 processes $\times 32$ threads) in this study.

\section{Results and Discussions}

At first of the simulation results, we show the case without interplanetary magnetic field (IMF). Figure 4 show the temporal evolutions of electron number density distributions normalized by the solar wind at upstream. The magnetosphere expands due to the line dipole fields excited by the applied current and the electron number density distributions vary with time as shown in Figs. 4 (a)(c). In Fig. 4 (c), the electron number density increase at the upstream of applied current like a bow shock formation. Moreover the wake region appears and the density decreases at the downstream of applied current. These are the result of reflection and deflection due to the interaction of plasma particles to the dipole field.

Figure 5 show the computational grids drawing on the electron number density distributions at $t \omega_{\mathrm{ce}}=60$ (which corresponds to Fig. 4 (c)). As mentioned above, in general, the grid width should be set to shorter than local Debye length in full PIC simulation. Debye length is inversely proportional to the square root of electron number density. Therefore, in the present AMR-PIC simulation code, we set a criterion that a higher level layer is locally created with the half grid size and time step interval when the local Debye length becomes shorter than the grid width of current layer. In Fig. 5, the spatial grids become fine (Lv.3) where the electron number density increases and become coarse (Lv.1) where that decreases compared to the base grids (Lv.2). These results correspond to the high density and wake region in Fig. 4 (c). We could confirm that hierarchical systems with fine and coarse grids are adaptively and locally created or deleted with the AMR scheme depending on the local variation of plasma condition.

In order to focus on the difference of solar wind flow field due to IMF direction, we examined the cases with $+Y$ and -Y direction IMF. Figures 6 (a) and (b) show the electron number density distributions and magnetic field lines at $t \omega_{\text {ce }}=60$ with $+Y$ direction and $-Y$ direction IMF, respectively. These contour level are the same as Fig. 4 (a). In the case with IMF, two magnetic neutral points appear because of the superposition of IMF and dipole field 


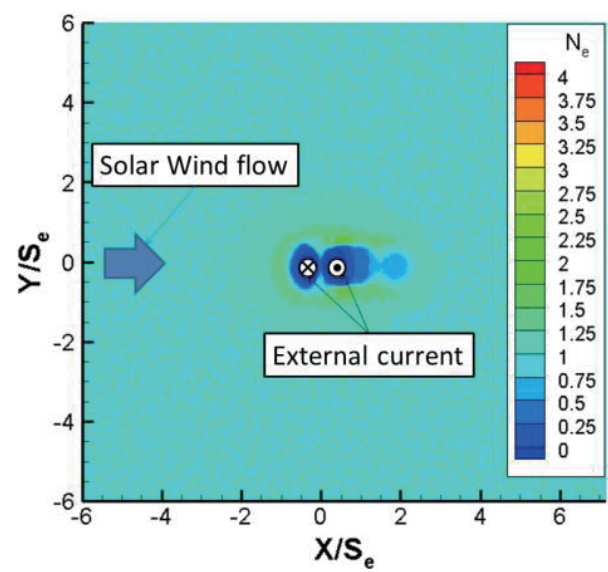

(a) $t \omega_{\text {ce }}=15$

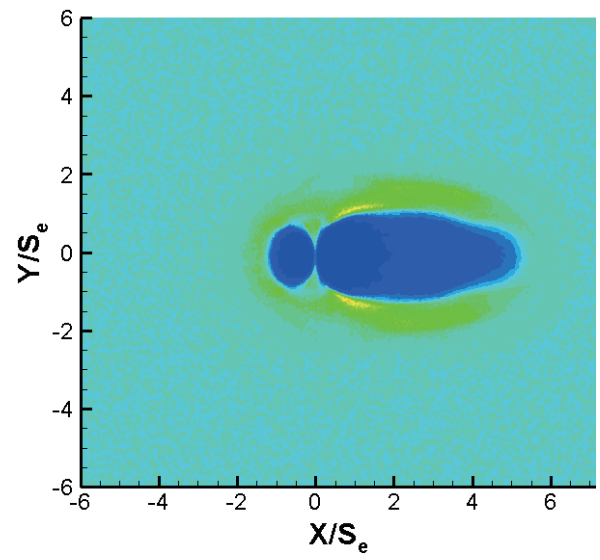

(b) $t \omega_{\mathrm{ce}}=30$

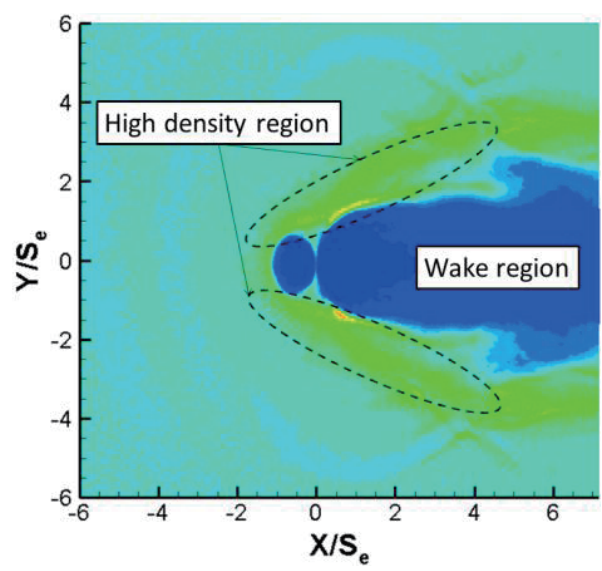

(c) $t \omega_{\mathrm{ce}}=60$

Fig. 4 Temporal evolutions of electron number density distribution at each time normalized by electron cyclotron frequency (a)-(c) without IMF case.

as shown in these figures. The electron number density distribution and the magnetospheric configuration depend strongly on the direction of IMF because the positions of magnetic neutral points change due to the direction of IMF. Such magnetic neutral points can be also found on the earth magnetosphere.

It is possible to appear the Alfven bow shock formation by applying IMF. Therefore, in order to examine the shock wave structures in details, we focus on the center

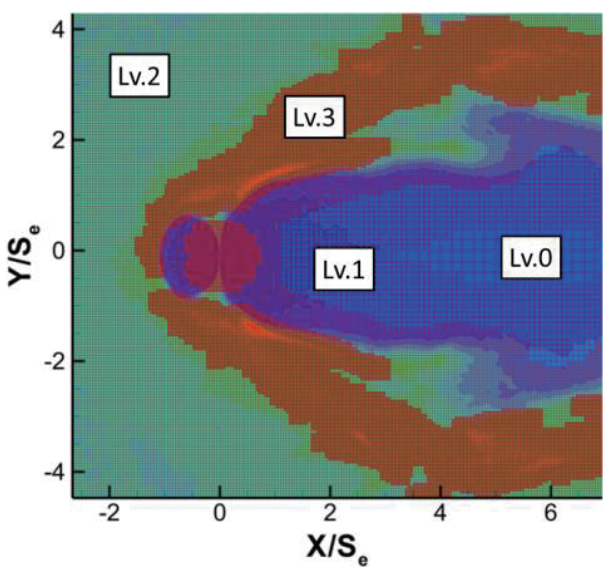

Fig. 5 Computational grids due to the adaptive mesh refinement drawing on electron number density distribution at $t \omega_{\text {ce }}=$ 60 (which corresponds to Fig. 4 (c)): Lv.0 (coarse grid) to Lv.3 (fine grid).

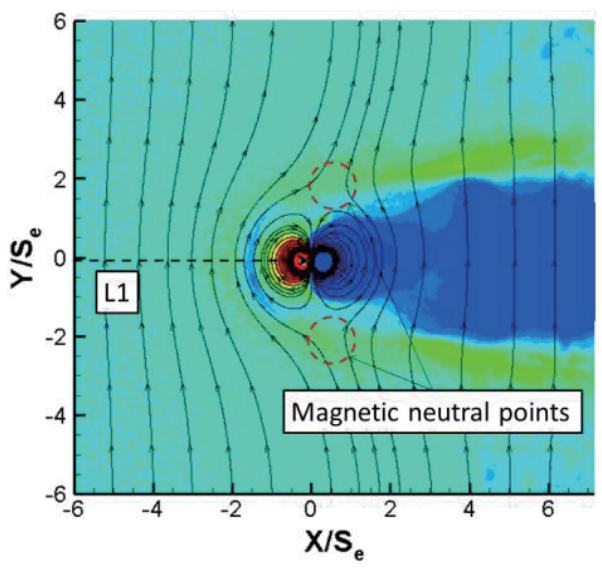

(a) Case with $+\mathrm{Y}$ direction IMF

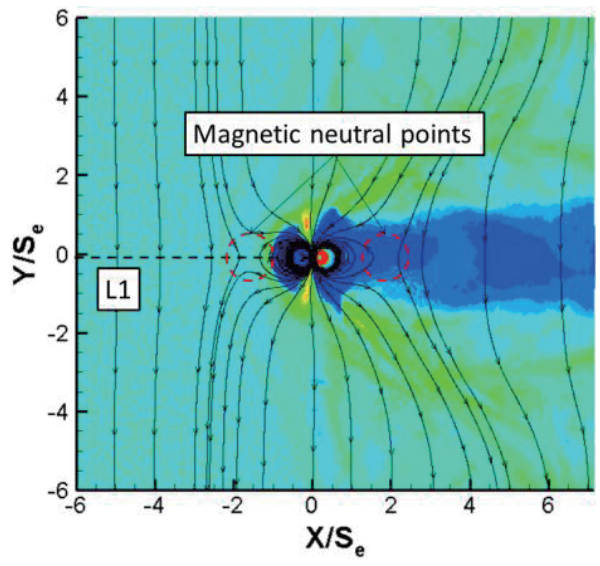

(b) Case with $-\mathrm{Y}$ direction IMF

Fig. 6 Electron number density distributions and magnetic field lines at $t \omega_{\mathrm{ce}}=60$ with IMF case.

line along L1 as shown in Fig. 6. Figures 7 (a) and (b) show the one dimensional distributions of Alfven Mach number $\left(M_{\mathrm{A}}\right)$, flow velocity $\left(V_{\mathrm{x}}\right)$ and Alfven speed $\left(V_{\mathrm{A}}\right)$ along L1 line. In the case with $+Y$ direction IMF of Fig. 7 (a), plasma flow at upstream is super-Alfvenic and the Alfven 


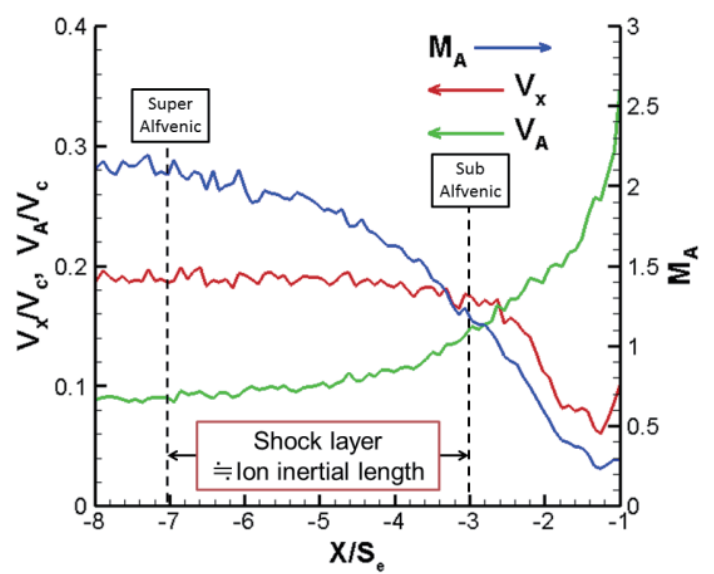

(a) Case with $+\mathrm{Y}$ direction IMF

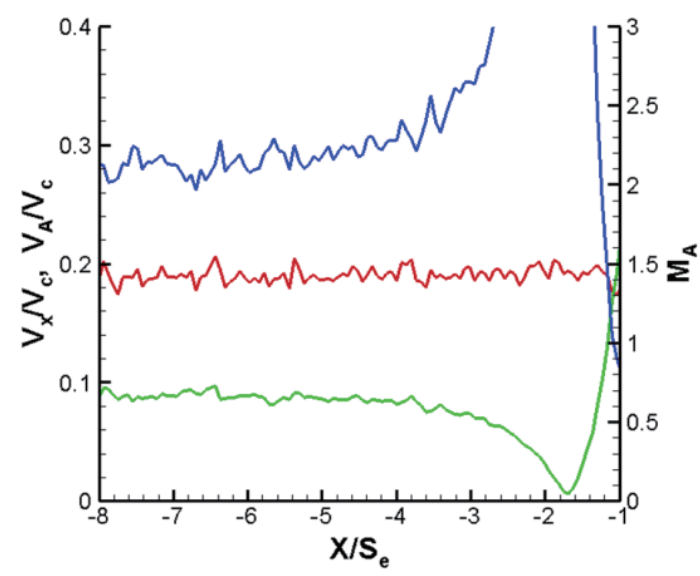

(b) Case with $-Y$ direction IMF

Fig. 7 One dimensional distributions of Alfven Mach number, flow velocity and Alfven speed along L1 line as shown in Fig. 6.

Mach number is about 2. However, the Alfven Mach number decreases and the solar wind is led to sub-Alfvenic flow toward the dipole center. Here, the Alfven shock layer is nearly equal to ion inertial length of solar wind. On the other hand, in the case with -Y direction IMF of Fig. 7 (b), such a shock wave structure cannot be observed because the magnetic neutral points appear in front of the dipole and the local Alfven speed decreases toward the dipole center. Because of that, the Alfven Mach number increases steeply in front of the dipole.

\section{Concluding Remarks and Future Plans}

The interaction of solar wind with kinetic scale magnetosphere around a magnetic sail was examined by using a parallelized two-dimensional AMR-PIC simulation code. As simulation results, it was shown that the direction of interplanetary magnetic field depend strongly on the magnetospheric configuration including electron dynamics and Alfven bow shock structure. In these results, the simulations including an electron inertial effect can be conducted and we can clarify the electron dynamics such as distributions of electron number density in the interaction of solar wind with kinetic scale magnetosphere by using newly developed AMR-PIC codes although it was never possible by conventional hybrid PIC codes. Although more validation studies are needed, the AMR-PIC code shows the worth of development for multi-scale simulations.

For the simulations with parameters of real space plasmas as a future plan, the computational performance of AMR-PIC code should be improved because many more grids and particles are needed. Therefore we should overcome the computational load imbalance problem between sub-domains corresponding to each processors associated with the process parallelization. In the AMR-PIC code, the difficulty is the load balancing between processors because the number of spatial grids and particles belonging to each sub-domain is not always constant due to the AMR procedure. In order to resolve the problem, we have already developed the load balancing technique called Dynamic Domain Decomposition (DDD) [9]. This technique divides whole computational domain into sub-domains of each processes dynamically so that the computational cost of spatial grids and particles becomes almost the same between the processes. We will adopt the DDD technique to the simulation with real parameters of space plasmas in future works.

We are particularly interested in the plasma phenomena occurring at the boundary region such as magnetic field reconnection point and shock structure as well as plasma turbulence. We will analyze such phenomena from a view point of multi-scale coupling between electrons and ions. By using the AMR-PIC code, we can simulate such waveparticle interactions with computer resources much less than ever used, which is one of the advantages of adopting the AMR scheme in a PIC code. Finally we will link these simulation results by the AMR-PIC code to the estimation and improvement of thrust performance of a magnetic sail.

\section{Acknowledgement}

This work was supported by the Japan Science and Technology Agency, Core Research for Evolutional Science and Technology (JST/CREST) under the project "High Performance Computing for Multi-Scale and MultiPhysics Phenomena". Also, in this research work, computations were performed using the KDK system of RISH, Kyoto University.

[1] R.M. Zubrin et al., J. Spacecraft Rockets 28, 197 (1991).

[2] R.M. Winglee et al., J. Geophys. Res. 105, 21067 (2000).

[3] H. Yamakawa et al., Acta Astronautica 59, 777 (2006).

[4] K. Fujita, J. Space Technol. Sci. 20, 26 (2004).

[5] Y. Kajimura et al., J. Propul. Power 28, 652 (2012).

[6] C.K. Birdsall and A.B. Langdon, Plasma Physics via Computer Simulation (Reprinted), (Taylor \& Francis, New York, 2005).

[7] H. Usui et al., Procedia Comp. Sci. 4, 2337 (2011).

[8] K. Fujimoto, J. Comput. Phys. 230, 8508 (2011).

[9] Y. Yagi et al., Proc. JSST2012 International Conference on Simulation Technology, Sep.27-28, Kobe, 587 (2012). 